\title{
Reduzindo a Variabilidade das Decisões de Roteamento em Redes em Malha Sem Fio
}

\author{
Bruno Silva, Diego Passos e Célio Albuquerque \\ ${ }^{1}$ Instituto de Computação - Universidade Federal Fluminense (UFF) \\ Niterói - RJ - Brazil \\ bsilva@id.uff.br, dpassos@ic.uff.br, celio@ic.uff.br
}

\begin{abstract}
Wireless mesh networks are multi-hop wireless networks which are useful in situations where there is little or no network infrastructure. Recently, those networks have been proposed for scenarios of Smart Grids and Internet of Things. The variability in routing metrics, which causes frequent changes in path choice, is a challenge in these networks. In this article, we propose a method, called Hypothesis Testing Estimator (HTE), for estimating the frame delivery probability of a link based on the concept of hypothesis testing. In this method, the estimate is fixed until the link behavior in the recent past significantly deviates from the expectation. The method was evaluated using simulations whose results show a significant reduction in the variability of the link quality estimates.
\end{abstract}

Resumo. Redes em malha sem fio são redes sem fio de múltiplos saltos úteis em situações em que há pouca ou nenhuma infraestrutura disponível. Recentemente, essas redes têm sido propostas para cenários de Smart Grids e Internet das Coisas. A variabilidade das métricas de roteamento, que ocasiona frequentes mudanças de rotas, é um desafio nessas redes. Neste artigo é proposto um método, chamado Hypothesis Testing Estimator (HTE), para estimar a probabilidade de entrega de quadros de um enlace baseado no conceito de teste de hipóteses. Nele, mantém-se uma estimativa fixa até que o comportamento do enlace no passado recente desvie significativamente do esperado. $O$ método foi avaliado através de simulações, cujos resultados mostram uma redução significativa na variabilidade das estimativas de qualidade dos enlaces.

\section{Introdução}

Redes em Malha sem Fio (ou WMNs, do inglês Wireless Mesh Networks) são redes sem fio de múltiplos saltos com aspectos particulares que permitem a cobertura de grandes áreas geográficas com pouca ou nenhuma infraestrutura prévia, e com baixo custo de implantação e manutenção [Akyildiz e Wang 2005]. Seu uso, hoje, não está restrito a esforços acadêmicos. Além de sua utilização em comunidades para acesso compartilhado à Internet, as WMNs têm sido usadas recentemente em novas aplicações, como o uso de RF-MESH na AMI (Advanced Metering Infrastructure) de Smart Grids [Rolim et al. 2015, Rolim et al. 2017]. Nós em uma WMN podem ser divididos em dois grupos: roteadores mesh e clientes mesh. Roteadores mesh são estáticos e funcionam como o backbone da rede. Pode haver também roteadores mesh especiais atuando como gateways com a responsabilidade de conectar a WMN a outras redes (i.e., a Internet). 
Apesar de seus muitos benefícios e vantagens potenciais, as WMNs ainda enfrentam obstáculos que prejudicam seu desempenho e, assim, restringem sua popularidade. Entre os principais desafios, pode-se citar a necessidade de aumentar a reutilização espacial através da redução da interferência intrafluxo e interfluxo [Passos 2013], um problema normalmente atacado sob perspectiva de roteamento [Laufer et al. 2009, Passos 2013].

O roteamento é, de fato, um dos mecanismos mais básicos para a operação de WMNs [Akyildiz e Wang 2005] e, assim, um dos focos principais de pesquisa nesta área. Mesmo outros mecanismos, à primeira vista não relacionados, são frequentemente estudados sob o prisma de roteamento. Como exemplos, tem-se esquemas de adaptação de taxa [Laufer et al. 2009], reuso espacial do espectro [Passos 2013] e codificação de rede [Passos 2013].

Em geral, a dificuldade em selecionar boas rotas em redes em malha sem fio está em modelar e prever o desempenho de caminhos existentes com base em informações básicas que podem ser medidas a partir de enlaces sem fio. Esta previsão é feita através do que se convencionou chamar na área de métricas de roteamento. Tais métricas atribuem pesos para cada enlace da rede com base em várias características mensuráveis [Campista et al. 2008]. Além disso, métricas de roteamento também determinam como os pesos dos enlaces individuais são combinados, de forma aditiva [De Couto et al. 2005] ou de forma multiplicativa [Passos et al. 2011], para avaliar a qualidade de uma rota completa.

A qualidade da seleção de rotas depende, portanto, da qualidade da métrica de roteamento. No entanto, esta qualidade também depende da acurácia das medidas usadas para calcular as características do enlace [Passos e Albuquerque 2012]. Outro fator associado com o desempenho do roteamento é a estabilidade da seleção de rotas. Protocolos e métricas que resultam em trocas mais frequentes de rotas, em geral apresentam pior desempenho [Passos et al. 2011, Passos 2013]. A instabilidade de roteamento pode não somente resultar no uso frequente de rotas subótimas, mas também levar à perda de pacotes ocasionada por loops [Yang et al. 2005]. Além disso, trocas de rotas desnecessárias podem influenciar negativamente o controle de congestionamento do TCP ao mudar abruptamente o RTT (Round Trip Time) de um caminho fim-a-fim, uma informação fundamental para o funcionamento apropriado deste mecanismo. Como exemplo mais concreto, em [Passos 2013] mostra-se que, mesmo em um cenário sem mobilidade dos nós, a variabilidade na seleção de nós em um protocolo de roteamento de estado de enlaces ocasionou quedas de até $30 \%$ no desempenho da rede.

Por essas razões, neste artigo, argumenta-se que há espaço para otimizações relacionadas à estabilidade de roteamento. Com isso em mente, propõe-se um novo método, chamado Hypothesis Testing Estimator (HTE), para estimar a probabilidade de entrega de um quadro em um enlace sem fio, uma informação que é comumente usada como base para calcular métricas de roteamento baseadas em qualidade [Campista et al. 2008].

Convencionalmente, este valor é estimado através do envio de uma série de quadros especiais, chamados probes, e do posterior processamento dos que são recebidos por nós vizinhos. Como será discutido brevemente neste trabalho, esta estimativa oscilará em torno da verdadeira probabilidade de entrega, o que introduzirá variabilidade no valor da métrica de roteamento e, desse modo, nas decisões de roteamento. 
Por outro lado, a proposta deste trabalho, baseada em teste de hipóteses, mantém um valor fixo para a estimativa de probabilidade de entrega até que uma sequência recente de probes desvie significativamente do comportamento esperado do enlace. Este trabalho mostra que o HTE é muito eficaz na filtragem de variações comuns de curto prazo associadas à estimativa tradicional empregrada por métricas de roteamento, ao mesmo tempo em que permite a detecção de mudanças na probabilidade de entrega assim como a convergência da estimativa para o novo valor.

Para avaliar o desempenho do HTE, foram conduzidas simulações numéricas e simulações de rede em um cenário com nós estáticos. Os resultados mostram que o HTE é, de fato, capaz de reduzir significativamente a variabilidade das estimativas de qualidade dos enlaces sem fio. Além disso, os resultados apontam espaço de melhora no HTE no que tange a detecção mais rápida de mudanças no comportamento do enlace. Embora o HTE seja usado neste trabalho para estimar a probabilidade de entrega de quadros, vislumbram-se cenários nos quais ele possa ser usado em outras aplicações com características similares, i.e., a necessidade de estimar os parâmetros de um sistema com baixa variabilidade.

O restante deste trabalho está organizado da seguinte forma. A Seção 2 discute os trabalhos relacionados. A Seção 3 descreve o HTE e suas diferenças para alternativas atualmente usadas. Na Seção 4 a proposta é avaliada por meio de simulações numéricas e de rede. Finalmente, a Seção 5 apresenta as conclusões.

\section{Trabalhos Relacionados}

A maior parte das métricas de roteamento para redes sem fio baseadas em qualidade atribui pesos para enlaces com base, direta ou indiretamente, na probabilidade de entrega dos quadros [Campista et al. 2008, De Couto et al. 2005, Draves et al. 2004, Passos et al. 2006]. Nesta seção, fornecemos uma breve visão geral desta literatura.

\subsection{Métricas de Roteamento Clássicas}

A métrica ETX (Expected Transmission Count) [De Couto et al. 2005] é uma métrica de roteamento que avalia enlaces de acordo com o número esperado de transmissões (e retransmissões) necessárias para entregar um pacote com sucesso. A métrica consegue modelar este aspecto de desempenho dos enlaces ao transmitir periodicamente probes e contar a perda de pacotes em ambas as direções de cada enlace.

Como probes são enviados periodicamente, se um probe não é recebido quando esperado, o nó receptor infere que este foi perdido e registra a perda de acordo. Probes também podem incluir números de sequência para auxiliar na identificação de perdas. Com base nas perdas registradas para os últimos probes enviados por um nó vizinho, cada nó receptor é capaz de computar uma estimativa para a probabilidade de entrega de quadros de um enlace. Como proposto originalmente, o ETX considera uma janela dos últimos $w$ probes. A probabilidade de entrega de quadros é, então, estimada como a razão entre o número de probes recebidos e o tamanho da janela. À medida que mais probes são recebidos ou perdidos, o nó receptor move sua janela e recalcula a estimativa da probabilidade de entrega de quadros. Esse procedimento permite ao ETX considerar um passado recente (o que é considerado recente é configurado ao manipular-se $w$ ). 
Outras formas de estimar a probabilidade de entrega de quadros incluem utilizar uma Média Móvel Exponencialmente Ponderada (EWMA - do inglês Exponentially Weighted Moving Average). Usando um fator de ponderação $\alpha$, a informação sobre a recepção ou perda de probes mais antigos tem seu peso diminuído exponencialmente. A principal vantagem do uso da EWMA em relação ao método original da janela deslizante é o pouco estado armazenado por enlace. De toda maneira, o comportamento a longo prazo das estimativas resultantes de ambos os métodos é similar.

De acordo com a notação usada em [De Couto et al. 2005], a razão entre o número de probes recebidos e o tamanho da janela $w$ (ou a saída da EWMA) é chamada $d_{r}$. Cada nó de origem transmite em seus probes os valores de $d_{r}$ correspondentes aos probes recebidos de seus vizinhos. Nós receptores então armazenam este valor como $d_{f}$. Assim, esse processo converge para uma situação na qual cada nó tem estimativas de probabilidade de entrega de quadros em ambas as direções, tanto para o envio quanto para o recebimento, para todos os seus enlaces. A multiplicação desses valores $\left(d_{f} \times d_{r}\right)$ é usada como uma aproximação da probabilidade de entrega de uma transmissão unicast completa bemsucedida no enlace (i.e., incluindo a transmissão do quadro ACK correspondente). O ETX do enlace é o recíproco deste valor:

$$
E T X=\frac{1}{d_{f} \times d_{r}}
$$

Sempre que o nó recebe um novo probe ou infere uma perda, $d_{f}$ e $d_{r}$ são atualizados e o ETX para o enlace é recalculado. O custo de uma rota é definido como a soma dos pesos dos enlaces que a compõem, com os menores valores representando melhores rotas.

Os autores da métrica ETT (Expected Transmission Time) [Draves et al. 2004] observam que em várias tecnologias, os enlaces sem fio podem empregar taxas de transmissão diferentes. Assim, eles propõem que a taxa de transmissão também deve ser considerada ao medir a qualidade do enlace. As probabilidades de entrega de quadros são calculadas usando o mesmo método empregado pela métrica ETX, mas o peso do enlace é calculado como o ETX multiplicado pelo atraso de transmissão de um quadro de tamanho fixo $s$. Assim, o ETT de um enlace corresponde a uma estimativa para o tempo necessário para enviar um pacote com um tamanho fixo pelo enlace, considerando o número esperado de retransmissões até o sucesso.

Outra métrica de roteamento que usa a probabilidade de entrega do quadro é a métrica ML (Minimum Loss) [Passos et al. 2006]. No entanto, contrariamente ao ETX e ETT que utilizam essas informações para estimar retransmissões e atrasos, respectivamente, ML a utiliza diretamente como o peso do enlace. Para calcular o custo total de um caminho, a métrica ML multiplica os pesos de cada enlace individual na tentativa de aproximar a probabilidade de entrega fim-a-fim.

Outros exemplos de métricas de roteamento que usam a probabilidade de entrega de quadros incluem WCETT [Draves et al. 2004] e mETX [Koksal e Balakrishnan 2006]. 


\subsection{Métricas de Roteamento que Consideram Variabilidade}

Há também na literatura alguns trabalhos que consideram métricas de roteamento especificamente voltadas para o objetivo de reduzir a variabilidade nas decisões de roteamento [Boushaba et al. 2013, Chen e Lee 2005, Dube et al. 1997, Rubin e Liu 2003].

A métrica Local Node Stability-based Routing (LNS) [Boushaba et al. 2013], por exemplo, baseia-se na noção de que rotas formadas por enlaces com pouca variação em sua estimativa de qualidade possuem menores taxas de perda de pacotes e atraso. Para diminuir a variabilidade na seleção de rotas, a LNS calcula um índice de estabilidade para cada enlace que forma a WMN e, com base nesses índices, atribui um valor de estabilidade para os nós. Cada nó transmite seu valor de estabilidade para seus vizinhos e, com base nesses valores recebidos, são calculadas as tabelas de roteamento.

O cálculo da Métrica de qualidade do enlace (LQM) é feito com base na Taxa de Interferência (IR) e no Nível de Congestionamento (CL). Os valores de LQM de um enlace, atual e pregresso, são então usados para calcular o índice de estabilidade do enlace. O índice é inicialmente ajustado para 0 (quando o LQM é menor do que um limiar prédefinido) ou 1 (quando maior) e, então, tem seu valor ajustado a cada nova análise de acordo com um algoritmo proposto pelos autores. $\mathrm{O}$ algoritmo divide o índice por 2, caso o LQM tenha degradado desde a última análise, ou adiciona 2, caso tenha melhorado, e adiciona 1 , caso não tenha modificado desde a última verificação. Se o LQM degradar a um valor abaixo do limiar, o índice recebe o valor 0 .

Com os índices dos enlaces definidos, é calculado o nível de estabilidade dos nós. As rotas são então escolhidas considerando-se os nós mais estáveis, ou seja, aqueles com os maiores valores de entropia (entre 0 e 1 ).

É importante destacar que as métricas clássicas (Seção 2.1) não levam em conta a minimização da variabiliade na estimativa de qualidade do enlace, enquanto que as métricas que consideram a variabilidade (Seção 2.2) avaliam apenas a variabilidade causada pelas alterações no tráfego e interferência na rede. Por outro lado, o HTE aborda essa variabilidade em um contexto mais fundamental, do ponto de vista dos próprios estimadores dos parâmetros de qualidade dos enlaces. Também por isso, o HTE é genérico o suficiente para ser aplicado a qualquer métrica baseada em qualidade de enlaces que use a probabilidade de entrega de quadros como informação base.

\section{O Método HTE}

Conforme discutido na Seção 2, métricas como o ETX usualmente utilizam algum tipo de média móvel (e.g., EWMA) dos resultados dos últimos $w$ probes para estimar a probabilidade de entrega de quadros. Esta estimativa, no entanto, tende a oscilar ao redor da probabilidade real, introduzindo variabilidade na métrica de roteamento. A Figura 1 ilustra esse comportamento. Ela mostra uma série temporal obtida a partir da aplicação de uma média móvel com uma janela deslizante dos últimos 10 probes sobre um trace sintético para $p=0,5$. Esse tamanho de janela é o mesmo proposto em [De Couto et al. 2005]. Embora as estimativas oscilem em torno do valor real de $p$, há uma variabilidade clara, com a estimativa atingindo o valor extremo 0 e aproximando-se do valor extremo $1 \mathrm{em}$ alguns pontos.

Pode-se argumentar que o aumento do tamanho da janela reduziria essa variabi- 


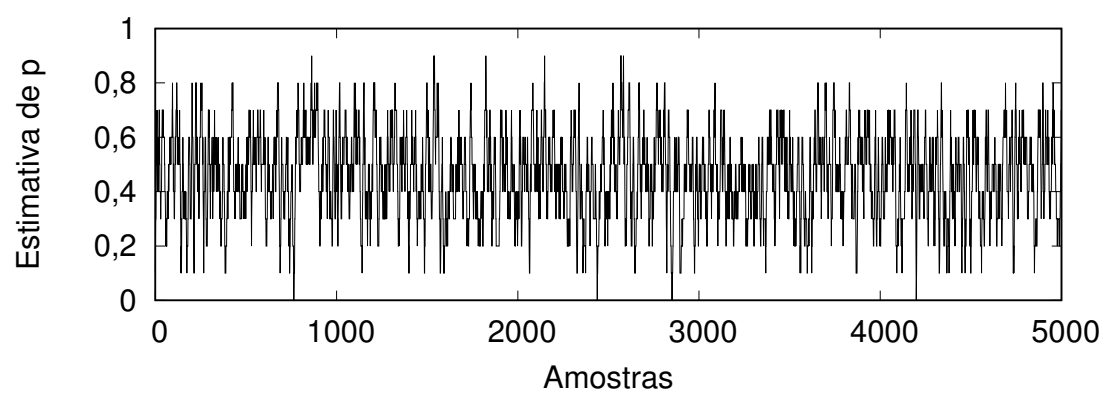

Figura 1. Série temporal demonstrando a evolução de uma estimativa para um trace $\operatorname{com} p=0,5$ e janela de tamanho $w=10$.

lidade. Todavia, a escolha do tamanho da janela inclui importantes tradeoffs. Aumentar o tamanho da janela também aumenta o intervalo de tempo considerado para estimar a probabilidade de entrega de quadros. Isso pode ser um problema porque o valor real da probabilidade pode mudar ao longo do tempo. Idealmente, métricas de roteamento detectariam e refletiriam essa mudança rapidamente. Com uma janela grande, esse processo se torna mais lento. Uma alternativa seria aliar o aumento do tamanho da janela com uma diminuição do intervalo entre probes (o que manteria relativamente curto o intervalo de tempo coberto pela janela). Esta solução também não é a ideal, uma vez que iria aumentar o overhead causado pela métrica de roteamento com os pacotes de controle. Assim, valores práticos para o tamanho da janela estão normalmente limitados ao redor de 50 probes [olsrd 2004]. Para o trace usado na Figura 1, no entanto, mesmo com uma janela $w=50$, as estimativas ainda oscilam entre 0,4 e 0,8 .

Na modelagem da estimativa da probabilidade de entrega usando probes, o resultado de cada probe, recepção ou perda, pode ser tomado como uma variável aleatória com distribuição de Bernoulli, fazendo com que o número de probes recebidos com sucesso seja uma variável aleatória com distribuição binomial de mesma probabilidade. Uma média móvel é aplicada nas amostras para estimar a probabilidade de entrega subjacente. Esta estimativa oscilará em torno da verdadeira probabilidade.

Para entender o funcionamento do HTE, considere novamente o trace usado na Figura 1 e suponha que, em um dado momento, a métrica de roteamento tem uma estimativa de $\hat{p}=0,55$, um valor relativamente próximo ao valor real de $p$. Com uma janela de tamanho $w=50$ probes, se essa estimativa for tomada como o valor real da probabilidade de entrega de quadros no enlace, a Função Densidade de Probabilidade de uma distribuição Binomial, mostrada na Figura 2, ilustra o que se pode esperar em termos dos números de probes recebidos com sucesso dentro da janela. Em $95 \%$ dos casos, o número de probes recebidos com sucesso na janela deve ficar entre 21 e 33 (indicado pela área hachurada na figura). Por outro lado, apenas 5\% do tempo pode-se esperar ter menos que 21 ou mais que 33 probes recebidos com sucesso em uma janela, assumindo que a probabilidade real seja $p=\hat{p}=0,55$.

Assim, dada uma estimativa da probabilidade de entrega de quadros, é possível definir uma região de comportamento esperado em torno do número esperado de sucessos. Se estivermos dispostos a trocar acurácia por precisão, esta região pode fornecer uma área de variação dentro do que pode ser considerado um estado esperado da janela con- 


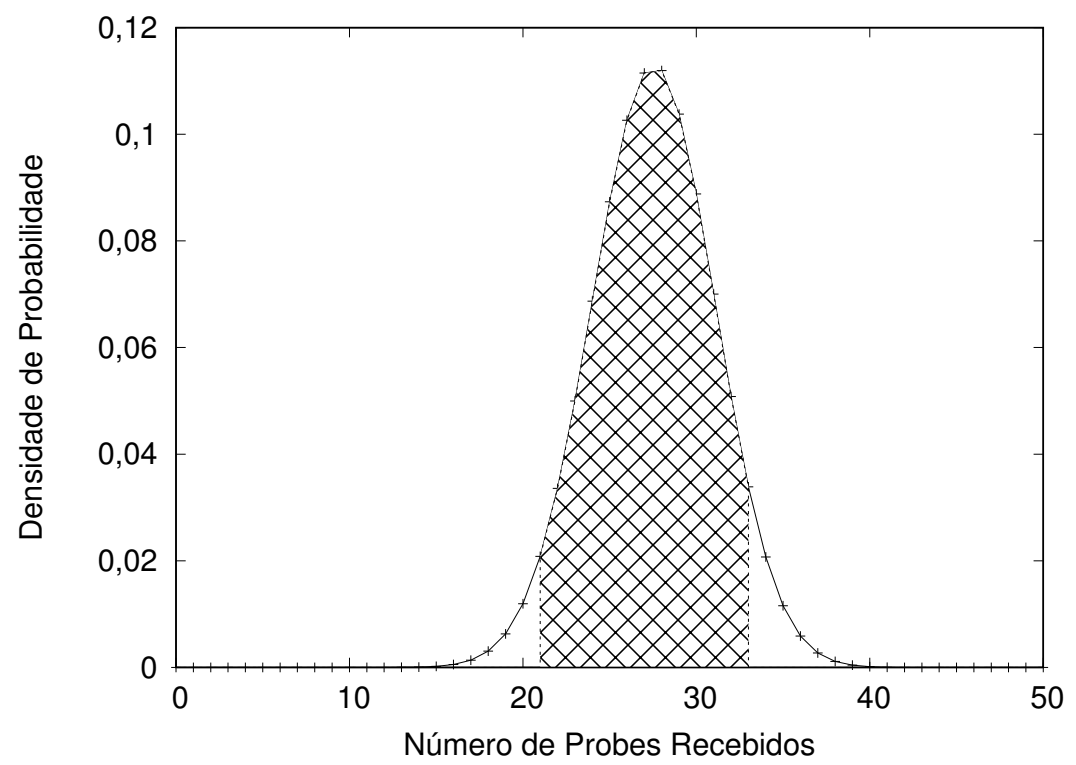

Figura 2. Distribuição do número esperado de probes recebidos com sucesso para $p=0,55 \mathbf{e} w=50$.

siderando a estimativa de probabilidade de entrega atual. Enquanto o número de probes recebidos se mantém nessa região, é razoável continuar a aceitar a estimativa atual como sendo uma representação justa da probabilidade de entrega de quadros. Por outro lado, quando um número anormalmente baixo ou elevado de probes recebidos é detectado, podemos considerar este evento como extremo. Isso pode indicar que a estimativa atual não está mais suficientemente próxima da probabilidade de entrega verdadeira (possivelmente devido a uma alteração na qualidade do enlace).

Esta é a ideia principal do HTE. Após obter uma estimativa inicial para a probabilidade de entrega de quadros, a estimativa é mantida até uma mudança significativa na probabilidade de entrega de quadros ser detectada. Na Figura 2, isso corresponderia a um estado da janela localizado fora da área hachurada. Note que isso é a basicamente um teste de hipóteses estatístico. A hipótese nula, $H_{0}$, assume que a probabilidade de entrega de quadros $p$ não mudou e, consequentemente, a estimativa $\hat{p}$ não deve ser al-

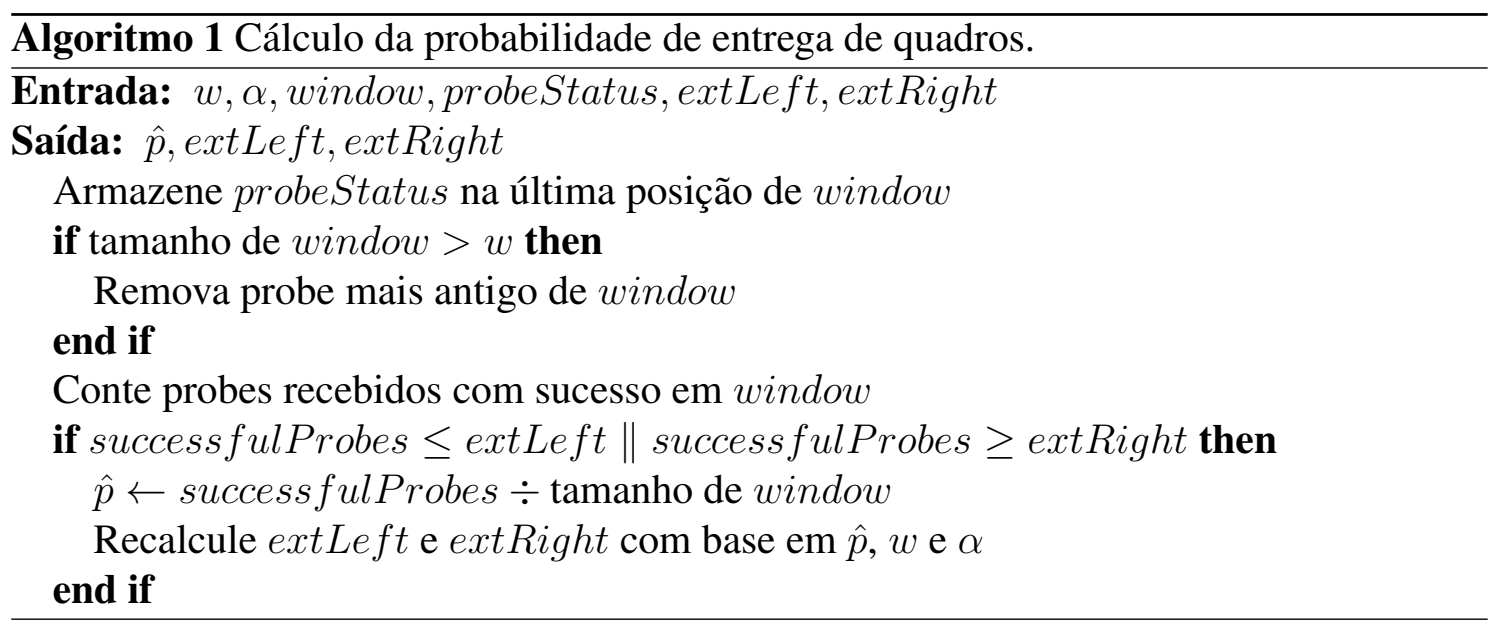


terada. A hipótese alternativa, $H_{1}$, considera que $p$ mudou significativamente, causando uma reavaliação da estimativa $\hat{p}$. Quando a probabilidade de entrega é modificada, a distribuição é recalculada e uma nova região de valor esperado é computada.

O Algoritmo 1 resume os passos executados pelo HTE a cada novo probe recebido ou perdido. A variável window é um vetor de valores booleanos de tamanho $w$ que registra se um probe foi recebido ou não. Após registrar o estado do probe na janela, o algoritmo contabiliza o número atual de probes recebidos com sucesso em success fulProbes e verifica se ele está entre extLeft e extRight (os valores extremos que definem a região de comportamento esperado). Se sim, a estimativa atual permanece inalterada e não é necessário processamento subsequente até o próximo probe. Caso contrário, atualizamos a estimativa de entrega de quadros e recalculamos os valores extremos com base na nova distribuição (que, por sua vez, é definida pela nova estimativa da probabilidade de entrega e pelo tamanho da janela $w$ ) e um nível de significância $\alpha$. Matematicamente, extLeft é definido como o maior número inteiro possível, tal que:

$$
\sum_{i=0}^{\text {extLeft }}\left(\begin{array}{c}
w \\
i
\end{array}\right) \times \hat{p}^{i} \times(1-\hat{p})^{w-i} \leq \frac{\alpha}{2} .
$$

Por outro lado, extRight pode ser calculado escolhendo o menor inteiro possível, de modo que:

$$
\sum_{i=e x t R i g h t}^{w}\left(\begin{array}{c}
w \\
i
\end{array}\right) \times \hat{p}^{i} \times(1-\hat{p})^{w-i} \leq \frac{\alpha}{2} .
$$

Deve-se notar que o cálculo dos valores extremos depende que $\hat{p}>0$. Sempre que um novo enlace é detectado pelo protocolo de roteamento, na ausência de informações adequadas, utilizamos arbitrariamente a estimativa inicial como $\hat{p}=0,25$. Esse valor é utilizado pois preferimos subestimar um enlace de melhor qualidade a superestimar um enlace de pior qualidade e, dessa forma, permitimos que a estimativa convirja gradualmente para a probabilidade real.

\section{Avaliação}

Nesta seção, o HTE é avaliado e analisado através de simulações numéricas e de simulações de rede. A primeira forma busca avaliar a estabilidade das estimativas retornadas pelo HTE sem que haja outros fatores influenciando na estimativa de qualidade. Já a segunda pretende demonstrar que, mesmo em face de fenômenos complexos inerentes à comunicação sem fio, como desvanecimento, o HTE consegue manter a estabilidade das suas estimativas.

\subsection{Simulações Numéricas}

As avaliações numéricas foram realizadas utilizando simuladores desenvolvidos na linguagem $\mathrm{C}$ que calculam a média móvel exponencialmente ponderada (EWMA) e o HTE através de teste de hipóteses. Ambos recebem como entrada um trace sintético contendo uma sequência de números ( 0 ou 1 ), que representam o recebimento ou não de probes utilizados pelo protocolo de roteamento, sendo o 1 indicador de recebimento e 0 o contrário. O trace utilizado nas simulações apresenta uma probabilidade real inicial $p=0,5$ e, após 5000 amostras, o valor de $p$ passa a valer 0,8 . 

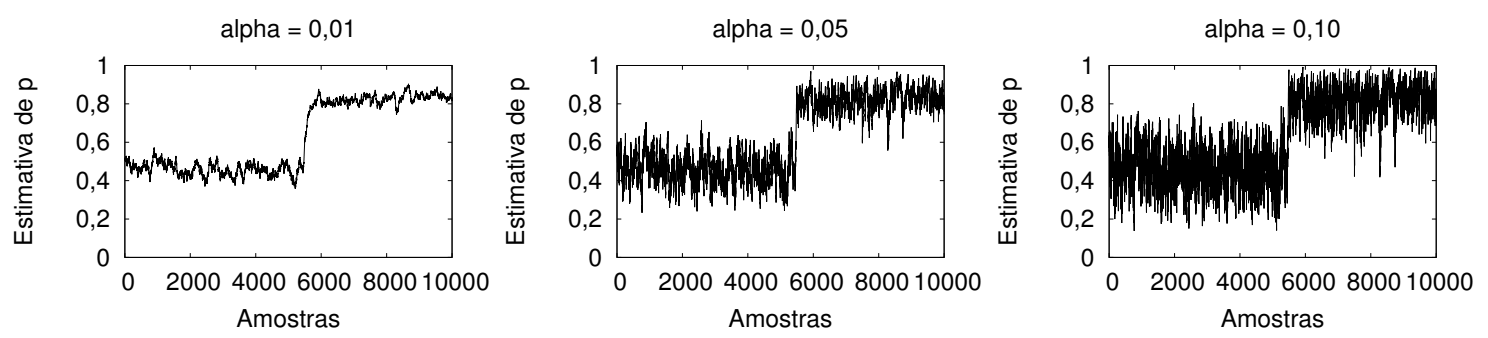

Figura 3. Gráficos da estimativa de probabilidade utilizando EWMA.

A Figura 3 apresenta os resultados das simulações numéricas utilizando o EWMA. Pelos gráficos, conforme esperado, podemos observar que a diminuição do valor $\alpha$ está diretamente ligada à diminuição na variabilidade da estimativa da probabilidade. No entanto, ainda assim ocorrem mudanças acentuadas no valor da estimativa num intervalo curto de amostras, mudanças essas que, em uma rede sem fio que utilizasse a estimativa como aferidora da qualidade de um enlace, poderiam ocasionar variabilidade na escolha de rotas.

Os resultados do HTE no mesmo trace, apresentados na Figura 4, mostram claramente que há uma diminuição na variação da estimativa da probabilidade. Esta diminuição fica evidente no gráfico com valores $w=170$ e $\alpha=0,01$, cuja estimativa de $p$ varia muito pouco e comparando-o com o EWMA com $\alpha=0,01$, percebe-se que o
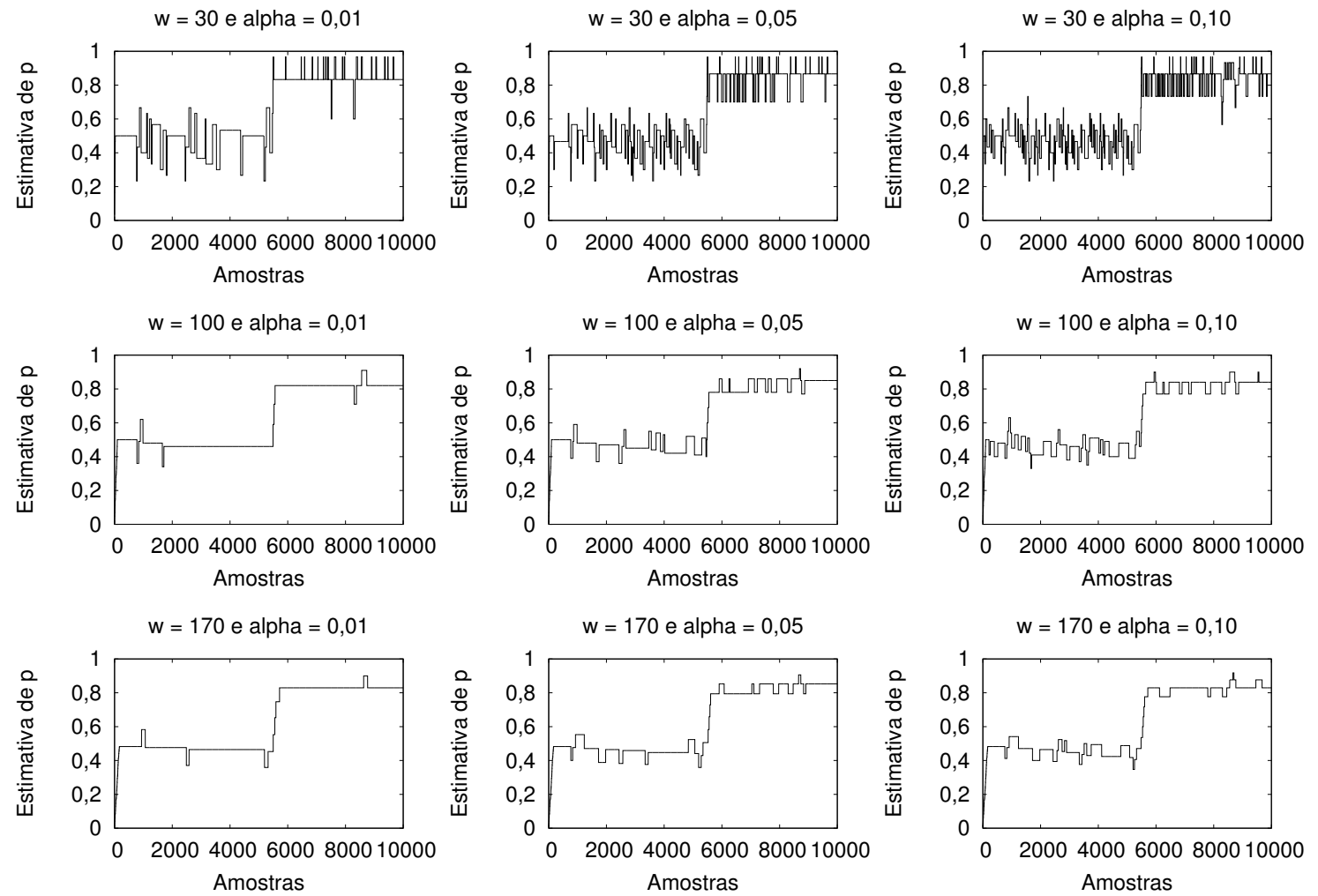

Figura 4. Gráficos da estimativa de probabilidade de entrega utilizando o HTE, variando-se $w \mathbf{e} \alpha$. 
HTE convergiu com pouquíssima variação. Além disso, o aumento do tamanho da janela para os mesmos valores de $\alpha$ aumenta o número de amostras a serem consideradas no teste de hipóteses e, portanto, a estabilidade das estimativas. Reduzir o valor de $\alpha$ também tem efeito similar, embora o tamanho da janela $w$ tenha mostrado maior influência na estabilidade da estimativa.

Podemos observar por esses resultados que, utilizando o HTE na estimativa da probabilidade, tem-se uma menor variabilidade em torno do valor real quando comparado ao método clássico, que utiliza uma média móvel exponencialmente ponderada. Deve-se notar que é possível obter tempos de convergência similares aos obtidos com o EWMA, utilizando-se parâmetros adequados. Para evidenciar isso, a Tabela 1 mostra o tempo de convergência dos estimadores quando ocorre a mudança de probabilidade no trace utilizado. Para computar esse tempo, foi utilizado como critério para determinar o final da convergência o instante em que cada método gera como saída a primeira estimativa a menos de $1 \%$ do valor real da probabilidade. É possível notar que mesmo resultando em menor variabilidade, os parâmetros utilizados para o HTE também alcançam tempos de convergência competitivos em relação aos parâmetros avaliados para o EWMA. Ademais, a análise da convergência revela um potencial aspecto de melhoria do HTE. Nos cenários avaliados, o início do processo de convergência demora mais para ocorrer no HTE. Dessa forma, um possível caminho de otimização desse aspecto seria a redução desse tempo inicial do processo de convergência.

\section{Tabela 1. Instante de término da convergência nas simulações numéricas, em número de amostras, para o EWMA e o HTE.}

\begin{tabular}{|c|c|c|c|c|c|}
\cline { 4 - 6 } \multicolumn{2}{c|}{} & \multirow{2}{*}{ EWMA } & \multicolumn{3}{|c|}{ HTE } \\
\cline { 4 - 6 } \multicolumn{2}{c|}{} & & \multicolumn{3}{|c|}{$w$} \\
\cline { 4 - 6 }$\alpha$ & $\mathbf{0 , 0 1}$ & 5667 & 5489 & 5561 & 5717 \\
\cline { 3 - 6 } & $\mathbf{0 , 0 5}$ & 5486 & 5490 & 5909 & 5611 \\
\cline { 2 - 6 } & $\mathbf{0 , 1 0}$ & 5478 & 5487 & 5645 & 5717 \\
\hline
\end{tabular}

\subsection{Simulações de Rede}

Como forma de estender e validar os resultados das simulações numéricas, nesta subseção, o HTE é analisado através de simulações de rede. O HTE foi implementado sobre o olsrd (versão 0.6.8) [olsrd 2004], uma versão de código livre do clássico protocolo de estado de enlace OLSR (Optimized Link State Routing) [Jacquet et al. 2001]. Essa implementação foi desenvolvida com base no código original do olsrd para a métrica ETX. Simulações foram executadas usando o simulador de redes ns-3 [ns3 2006] com o framework DCE [dce 2010], que permite o uso de aplicações e protocolos reais, sem a necessidade de adaptações ou mudanças em seus códigos fonte. Os modelos de perda de propagação utilizados nas simulações foram o Nakagami e o Log Distance, ambos com suas configurações padrão.

As simulações foram conduzidas com a versão original da implementação da métrica ETX baseada em números em ponto flutuante e que utiliza o EWMA. O HTE foi implementado como uma variação dessa implementação. Os parâmetros de configuração utilizados pelo HTE foram uma janela de tamanho $w=170$ e $\alpha=0,05$. O valor do 
$\alpha$ utilizado para o EWMA foi 0,01. Esses valores foram escolhidos com base nos resultados da Tabela 1, no qual os instantes de término da convergência correspondentes aos parâmetros selecionados são similares. Foi utilizada a configuração padrão fornecida pela implementação do olsrd em ambos os métodos, destacando-se que o intervalo padrão de envio de probes é de dois segundos. Na camada de enlace, os nós simulados usaram rádios IEEE 802.11b [IEEE 802.11 1997] com a taxa de transmissão fixada em $1 \mathrm{Mb} / \mathrm{s}$.

Como um primeiro experimento objetivando verificar o tempo de convergência, foi criada uma topologia na qual dois nós são posicionados inicialmente a uma distância de 125 metros um do outro. Após 420 segundos de simulação, o segundo nó é movido instantaneamente para uma distância de 165 metros do nó 1 . A partir desse momento, observa-se o tempo que os métodos levam para convergir à nova estimativa de probabilidade de entrega de quadros.

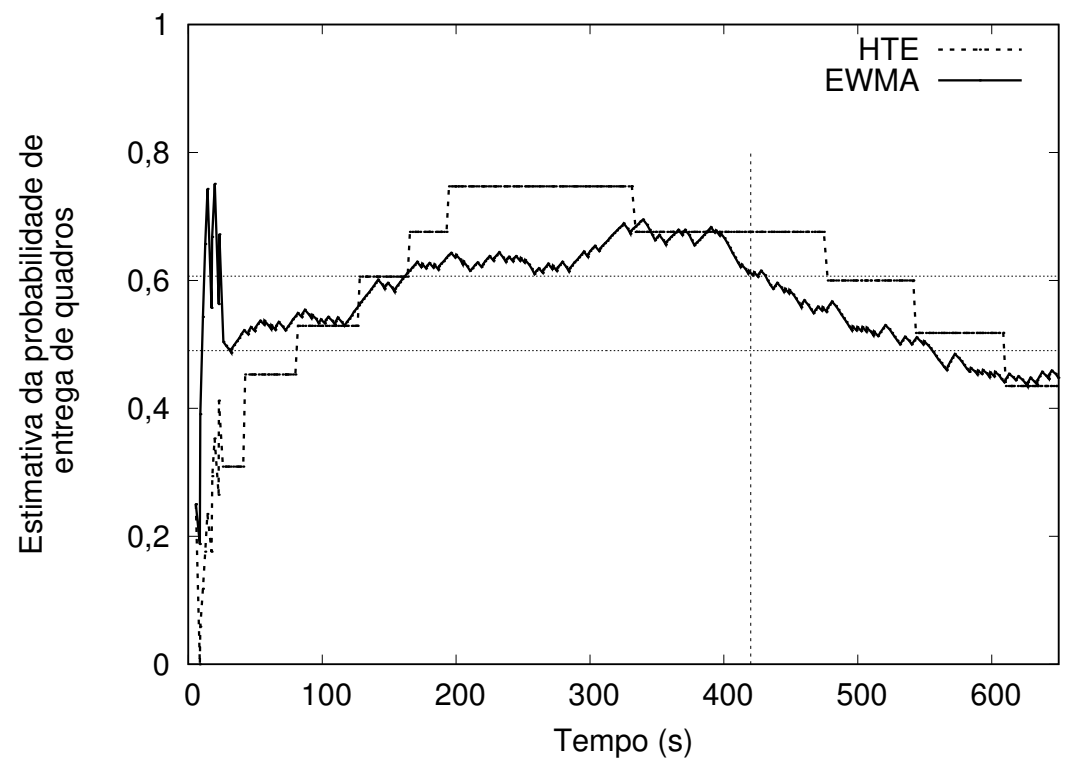

Figura 5. Verificação do tempo de convergência na estimativa da probabilidade de entrega entre o EWMA e o HTE em simulação de rede.

A Figura 5 apresenta o resultado dessa simulação. As duas linhas horizontais no gráfico representam o valor médio da probabilidade de entrega antes (superior) e após (inferior) a movimentação do segundo nó enquanto a linha vertical indica o momento em que o segundo nó é movido. Esses valores foram calculados através da média das estimativas do EWMA antes e depois da movimentação do segundo nó. O gráfico demonstra que tanto o EWMA como o HTE convergem para a nova probabilidade de entrega próximo ao instante 530 segundos.

Para avaliar o HTE em relação à variabilidade, utilizou-se uma topologia composta por 11 nós (Figura 6) dispostos de tal forma que existam duas rotas possíveis conectando os nós 0 e 1 (uma passando pelos nós da porção superior da topologia e outra utilizando os nós da porção inferior). O objetivo foi observar a evolução da estimativa de entrega de quadros pelo HTE para vários enlaces da topologia.

Os gráficos da Figura 7 mostram o comportamento de dois desses enlaces considerando a borda inicial e o meio do trajeto até o nó destino. A linha tracejada em cada 


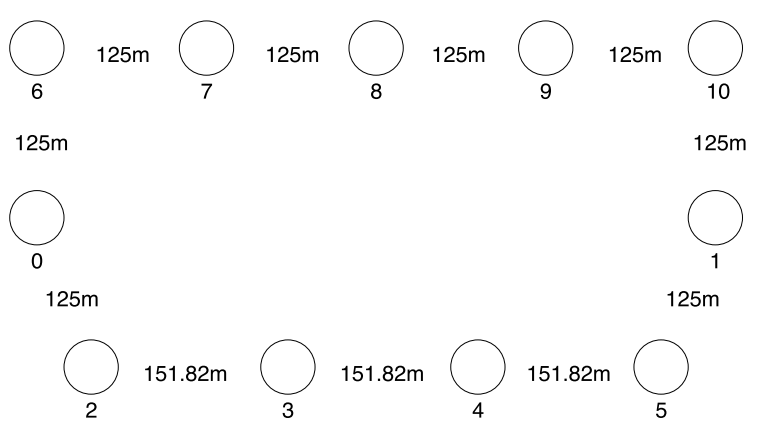

Figura 6. Topologia composta por 11 nós para verificação de variabilidade.

gráfico representa o valor da probabilidade de entrega de quadros subjacente de cada enlace. Assim como no experimento anterior, esses valores foram obtidos através da média das amostras resultantes da EWMA para toda a duração da simulação (700 segundos). Assim como nas simulações numéricas, os resultados mostram que a estimativa converge para valores próximos ao da probabilidade real, mas com baixa variabilidade durante o curso da simulação, mesmo em face de fenômenos complexos de propagação de rádio.
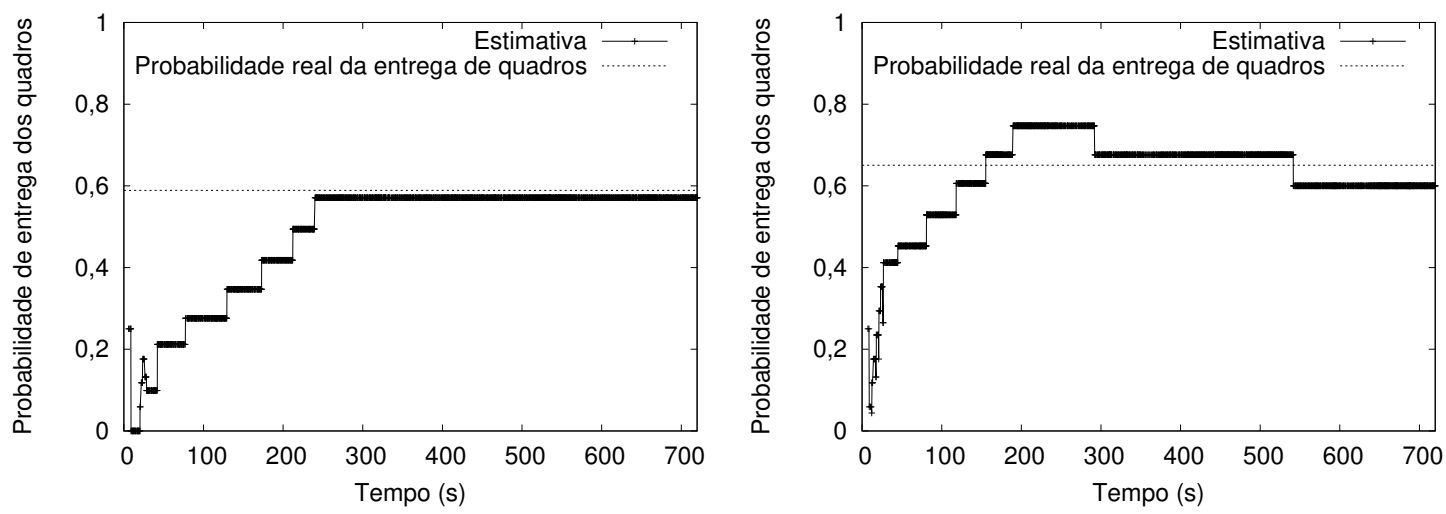

Figura 7. Estimativa da probabilidade de entrega de quadros pelo HTE para a borda do cenário (nó 0 em direção ao nó 2 ) e para o meio do cenário (nó $8 \mathrm{em}$ direção ao nó 9).

\section{Conclusão}

Dadas as características das redes em malha sem fio e sua utilidade em cenários nos quais uma infraestrutura não está disponível ou é inexistente, é importante avançar e melhorar as métricas e protocolos que permitem seu correto e eficiente funcionamento. $\mathrm{O}$ emprego dessas redes nos inovadores cenários de Smart Grids e de Internet das Coisas motiva a busca por soluções que extraiam maior desempenho dessas redes.

Este trabalho propõe uma abordagem inovadora para estimar a probabilidade de entrega de quadros de um enlace, uma informação importante que é comumente a base para métricas de roteamento baseadas em qualidade de enlaces. O HTE utiliza um teste de hipóteses estatístico para reduzir a variabilidade das decisões de roteamento e, consequentemente, para melhorar o desempenho da rede. No HTE, após uma estimativa inicial ser encontrada, seu valor é usado para definir uma região de comportamento esperado. Conforme novos pacotes probes são perdidos ou recebidos, o HTE verifica se o número 
de probes recebidos com sucesso na janela atual ainda está dentro desta região. Se esse for o caso, a estimativa atual permanece inalterada. Caso contrário, computa-se uma nova estimativa com base nos últimos probes recebidos e uma nova região de comportamento esperado é limitada. Este procedimento é bem eficaz na filtragem de variações de curto prazo desta estimativa, enquanto ainda fornece estimativas com boa acurácia.

Os resultados das simulações numéricas mostram que o HTE apresenta estabilidade na estimativa de probabilidade. Demonstram ainda que para diferentes tamanhos de janela $w$ e significância $\alpha, o w$ tem maior influência na variabilidade da estimativa. Já as simulações de rede reafirmam a estabilidade do HTE mesmo em face das variações introduzidas pela dinâmica da rede e apresentam tempos de convergência muito similares entre o EWMA $(\operatorname{com} \alpha=0,01)$ e o HTE para $w=170$ e $\alpha=0,05$.

Como trabalho futuro, planejamos estender a avaliação testando a sensibilidade do HTE a diferentes valores de $\alpha$ e $w$ e avaliar seu desempenho em cenários com mobilidade e/ou grande variabilidade nas condições do enlace. Pretende-se, ainda, trabalhar na otimização do tempo de convergência do HTE. A redução desse tempo é importante não apenas para acelerar a convergência inicial das estimativas de qualidade dos enlaces, mas também para a rápida transição para novos valores quando o desempenho do enlace de fato sofre mudanças. Uma otimização desse aspecto permitiria o emprego de parâmetros $w$ e $\alpha$ que preconizem a estabilidade das estimativas, mantendo a velocidade de convergência atual do HTE com parâmetros mais agressivos. Almeja-se também estudar a combinação do HTE com o algum outro mecanismo que seja capaz de lidar com variações nas estimativas de qualidade dos enlaces causadas pelas colisões de probes do protocolo de roteamento com pacotes de dados quando a rede se encontra sob altas cargas. Por fim, pretende-se realizar medições de desempenho de rede, para avaliar o efeito completo da proposta.

\section{Referências}

Akyildiz, I. F. e Wang, X. (2005). A survey on wireless mesh networks. IEEE Communications magazine, 43(9):S23-S30.

Boushaba, M., Hafid, A., e Gendreau, M. (2013). Local node stability-based routing for wireless mesh networks. Em 2013 IEEE Wireless Communications and Networking Conference (WCNC), pages 1950-1955.

Campista, M. E. M., Esposito, P. M., Moraes, I. M., Costa, L. H. M., Duarte, O. C. M., Passos, D. G., De Albuquerque, C. V. N., Saade, D. C. M., e Rubinstein, M. G. (2008). Routing metrics and protocols for wireless mesh networks. IEEE network, 22(1).

Chen, L. e Lee, C.-w. (2005). Neighbor stability routing in MANETs. Em 2005 IEEE Wireless Communications and Networking Conference, volume 4, pages 1964-1969.

dce (2010). DCE. https://www.nsnam.org/overview/projects/ direct-code-execution. Acesso em 27 Fev. 2016.

De Couto, D. S., Aguayo, D., Bicket, J., e Morris, R. (2005). A high-throughput path metric for multi-hop wireless routing. Wireless Networks, 11(4):419-434.

Draves, R., Padhye, J., e Zill, B. (2004). Routing in multi-radio, multi-hop wireless mesh networks. Em Proceedings of the 10th annual international conference on Mobile computing and networking, pages 114-128. 
Dube, R., Rais, C. D., Wang, K.-Y., e Tripathi, S. K. (1997). Signal stability-based adaptive routing (SSA) for ad hoc mobile networks. IEEE Personal communications, $4(1): 36-45$.

IEEE 802.11 (1997). Wireless LAN medium access control (MAC) and physical layer (PHY) specifications. IEEE Standard 802.11-1997.

Jacquet, P., Muhlethaler, P., Clausen, T., Laouiti, A., Qayyum, A., e Viennot, L. (2001). Optimized link state routing protocol for ad hoc networks. Em IEEE International Multi Topic Conference, 2001. IEEE INMIC 2001. Technology for the 21st Century. Proceedings., pages 62-68.

Koksal, C. E. e Balakrishnan, H. (2006). Quality-aware routing metrics for timevarying wireless mesh networks. IEEE Journal on selected areas in communications, 24(11):1984-1994.

Laufer, R., Dubois-Ferriere, H., e Kleinrock, L. (2009). Multirate anypath routing in wireless mesh networks. Em IEEE INFOCOM 2009, pages 37-45.

ns3 (2006). ns-3. https : / / www . nsnam. org. Acesso em 27 Fev. 2016.

olsrd (2004). Olsr.org wiki. http: / / www . olsr.org. Acesso em 29 Jan. 2016.

Passos, D. (2013). Flow-Based Interference-Aware Routing in Multihop Wireless Networks. Tese de Doutorado, Instituto de Computação, Universidade Federal Fluminense, Niterói, RJ, Brasil.

Passos, D. e Albuquerque, C. V. (2012). A joint approach to routing metrics and rate adaptation in wireless mesh networks. IEEE/ACM Transactions on Networking (TON), 20(4):999-1009.

Passos, D., de Albuquerque, C. V. N., Campista, M. E. M., Costa, L. H. M., e Duarte, O. C. M. (2011). Minimum loss multiplicative routing metrics for wireless mesh networks. Journal of Internet Services and Applications, 1(3):201-214.

Passos, D., Teixeira, D. V., Muchaluat-Saade, D. C., Magalhães, L. C. S., e Albuquerque, C. (2006). Mesh network performance measurements. Em International Information and Telecommunicatios Technologies Symposium (I2TS), pages 48-55.

Rolim, G., Passos, D., Albuquerque, C., e Moraes, I. (2017). Moskou: A heuristic for data aggregator positioning in smart grids. IEEE Transactions on Smart Grid.

Rolim, G., Passos, D., Moraes, I., e Albuquerque, C. (2015). Modelling the data aggregator positioning problem in smart grids. Em 2015 IEEE International Conference on Computer and Information Technology; Ubiquitous Computing and Communications; Dependable, Autonomic and Secure Computing; Pervasive Intelligence and Computing (CIT/IUCC/DASC/PICOM), pages 632-639.

Rubin, I. e Liu, Y.-C. (2003). Link stability models for QoS ad hoc routing algorithms. Em 2003 IEEE 58th Vehicular Technology Conference, volume 5, pages 3084-3088.

Yang, Y., Wang, J., e Kravets, R. (2005). Designing routing metrics for mesh networks. Em IEEE Workshop on Wireless Mesh Networks (WiMesh), pages 1-9. 